\title{
Processo Inflamatório em Mucosa de Reservatórios Ileais de Doentes Assintomáticos
}

\section{Inflammatory Activity of Pelvic Ileal Pouches Mucosa in Asymptomatic Patients}

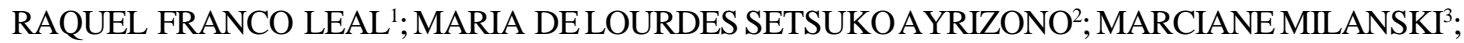 \\ JOÃO JOSÉFAGUNDES ${ }^{4}$; ANDRESSACOOPE ${ }^{3}$; SANDRO NUNES ÂNGELO $^{5} ;$ LÍCIOAUGUSTO VELLOSO $^{6}$; \\ CLÁUDIOSADDY RODRIGUES COY ${ }^{4}$
}

\begin{abstract}
${ }^{1}$ Mestre em Cirurgia, Residência Médica em Coloproctologia pela Universidade Estadual de Campinas (UNICAMP); ${ }^{2}$ Professor Doutor, Grupo de Coloproctologia - DMAD -UNICAMP; ${ }^{3}$ Pós-graduação em Clínica Médica pela UNICAMP;

${ }^{4}$ Professor Livre Docente, Grupo de Coloproctologia, DMAD-UNICAMP; ${ }^{5}$ Mestre em Cirurgia - Grupo de Coloproctologia, DMAD - UNICAMP; ${ }^{6}$ Professor Associado, Departamento de Clínica Médica, FCM-UNICAMP.
\end{abstract}

\begin{abstract}
LEAL RF; AYRIZONO MLS; MILANSKI M; FAGUNDES JJ; COOPE A; ÂNGELO SN; VELLOSO LA; COY CSR. Processo Inflamatório em Mucosa de Reservatórios Ileais de Doentes Assintomáticos. Rev bras Coloproct, 2008;28(4): 443-448.

RESUMO: A inflamação inespecífica do reservatório ileal (RI) após retocolectomia total é a complicação pós-operatória mais comum nos doentes operados por retocolite ulcerativa inespecífica (RCUI). Os mecanismos imunológicos envolvidos na etiologia não são totalmente conhecidos. Objetivo: Avaliar a ativação de STAT-1 e a expressão da citocina INF- $\gamma$ em mucosa de RI endoscópica e histologicamente normal, de doentes operados por RCUI e PAF, assintomáticos. Casuística e Método: Estudou-se 18 doentes submetidos à retocolectomia total com RI em "J", sendo nove com RCUI e nove com polipose adenomatosa familiar (PAF). Realizou-se biópsias da mucosa dos RI e de íleo terminal. As expressões de INF- $\gamma$ e a ativação de STAT-1 foram avaliadas por meio de imunoblot de extrato protéico total. Resultados: A ativação de STAT-1 foi maior em mucosa de RI de doentes operados por RCUI, quando comparada aos grupos PAF e Controle $(\mathbf{p}<0.05)$. A expressão de INF- $\gamma$ foi maior no grupo RCUI quando comparada ao grupo Controle (p<0.05), mas não em relação ao grupo PAF (p>0.05). Conclusão: Estes achados podem justificar a maior susceptibilidade dos doentes com RCUI à inflamação inespecífica do RI quando comparados aos portadores de PAF, sendo a principal via inflamatória similar à verificada na RCUI.
\end{abstract}

Descritores: Retocolite ulcerativa; polipose adenomatosa familiar; reservatório ileal; bolsa ileal; bolsite; citocinas.

\section{INTRODUÇÃO}

Retocolectomia total com confecção do reservatório ileal (RI) é o procedimento de escolha para doentes com retocolite ulcerativa inespecífica (RCUI) com intratabilidade clínica, e para aqueles com polipose adenomatosa familiar (PAF) que apresentam, em geral, mais que vinte pólipos retais. ${ }^{1,2,3,4,5,6}$ Entretanto, a inflamação inespecífica primária do RI ("bolsite") é uma das complicações mais comuns após este procedimento prin- cipalmente em RCUI, atingindo até $60 \%$ de ocorrência, enquanto que apenas $5 \%$ dos portadores de PAF a apresentam. ${ }^{7,8,9,10,11}$ A etiologia não é totalmente esclarecida e o diagnóstico é clínico, porém deve ser confirmado pelos achados endoscópicos e histológicos. ${ }^{12-22}$

A diferença da incidência de "bolsite" entre os doentes com RCUI e PAF tem levado alguns autores à hipótese de que possa haver similaridade entre esta complicação e a própria doença inflamatória intestinal de base. ${ }^{23,24}$

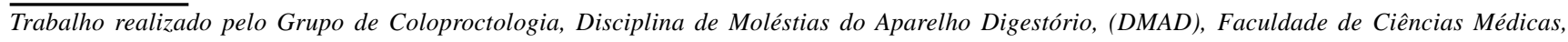
Universidade Estadual de Campinas (UNICAMP), Campinas, São Paulo, Brasil. Laboratório de Sinalização Celular, Departamento de Medicina Interna, Faculdade de Ciências Médicas, Universidade Estadual de Campinas (UNICAMP), Campinas, São Paulo, Brasil. 
As citocinas pró-inflamatórias e as vias de sinalização celular envolvendo o grupo de proteínas ativadoras de transcrição nuclear (STAT) apresentam papel importante na etiopatogenia da RCUI. Especificamente, níveis elevados de Interferon-ã (INF- $\gamma$ ) e STAT-1 foram detectados em amostras de mucosa intestinal de doentes portadores de doença inflamatória intestinal, particularmente em RCUI. ${ }^{25-30}$ No entanto, poucos estudos têm avaliado o processo inflamatório em mucosa de RI com ileíte do reservatório e nas condições de doentes assintomáticos ${ }^{7,31-37}$, e determinado se há correlação com a RCUI. . $^{38-42}$

Assim, com a finalidade de avaliar o processo inflamatório em mucosa de RI de doentes operados por RCUI e PAF com o trânsito íleoanal reconstruído, assintomáticos, determinou-se a expressão da citocina pró-inflamatória IFN- $\gamma$ e do fator de transcrição nuclear do TNF- $\alpha$, STAT-1, comparando-os nas condições de doença acima citadas, e em grupo controle.

\section{CASUÍSTICA E MÉTODOS}

Biópsias de mucosa de RI foram obtidas de nove doentes portadores de RCUI assintomáticos, com média de idade de 48,7 (31-63) anos, sendo 55,6\% do sexo feminino, e de nove portadores de PAF, também assintomáticos com média de idade de 33,8 (21-59) anos e 55,6\% do sexo feminino. O tempo de seguimento pósoperatório foi de 73,1 (24-168) meses. Os reservatórios ileais foram do tipo "J", e a arcada vascular do cólon direito foi preservada como complementação à irrigação sanguínea do íleo terminal. ${ }^{43}$ A mucosectomia foi realizada e a anastomose íleo-anal foi confeccionada manualmente. Os pacientes haviam sido submetidos à reconstrução de trânsito, com fechamento da ileostomia de proteção há mais de um ano. A ausência de ileíte do RI foi observada clinicamente, além da análise endoscópica e histológica realizada de acordo com o Índice de Atividade da Ileíte do RI, proposto por Sandborn e $\mathrm{col}^{20}$. O grupo controle foi composto por nove indivíduos com íleo-colonoscopia normal, sendo a média de idade de 40,9 (26-58) anos e 55,6\% do sexo feminino. As biópsias foram obtidas do íleo distal.

O estudo foi realizado pelo Grupo de Coloproctologia e Laboratório de Sinalização Celular da Universidade Estadual de Campinas e aprovado pelo comitê de ética local registrado sob $\mathrm{n}^{\circ}$ 543/2005 com adendo em 2007. Todos os pacientes assinaram o termo de consentimento informado.
Fragmentos de íleo do RI e de íleo terminal foram congelados em nitrogênio líquido, e armazenados a $-80^{\circ} \mathrm{C}$, no Laboratório de Sinalização Celular FCM UNICAMP, para posterior homogeneização em tampão de imunoprecipitado contendo $1 \%$ de Triton $\mathrm{X} 100,100 \mathrm{mM}$ de Tris (ph 7,4), 100mM de pirofosfato de sódio, $100 \mathrm{mM}$ de fluoreto de sódio, $10 \mathrm{mM}$ de EDTA, $10 \mathrm{mM}$ de vanadato de sódio, $2 \mathrm{mM}$ de PMSF e $0,1 \mathrm{mg} / \mathrm{ml}$ de aprotinina a $4^{\circ} \mathrm{C}$. O homogeneizado foi então centrifugado à $11.000 \mathrm{rpm}$ por 20 minutos. No sobrenadante foi determinada a concentração de proteína utilizando o método de Bradford ${ }^{44}$, e posteriormente preparou-se amostra contendo 100ìg para separação por SDS-PAGE. As amostras foram diluídas em tampão de Laemmli, contendo $100 \mathrm{mmol} / \mathrm{L}$ de DTT. Após rápida fervura, foram aplicadas em gel de poliacrilamida para separação por eletroforese (SDS-PAGE). As proteínas separadas em SDSPAGE foram transferidas para membrana de nitrocelulose, em aparelho de transferência da BIORAD. A membrana de nitrocelulose foi incubada "overnight" com 10ìl de anticorpo específico (antiIFN- $\gamma$, anti-STAT-1). A ligação do anticorpo a proteínas não-específicas foi minimizada pela pré-incubação da membrana de nitrocelulose com tampão de bloqueio (5\% de leite em pó desnatado; $10 \mathrm{mmol} /$ $\mathrm{L}$ de Tris; $150 \mathrm{mmol} / \mathrm{L}$ de $\mathrm{NaCl} ; 0,02 \%$ de Tween 20) por 1,5 hora. ${ }^{45,46}$

Os reagentes para separação das proteínas em gel de poliacrilamida e imunoblot foram da Bio-Rad Laboratories (Richmond, CA). Aprotinina, Triton X-100, Tween 20, glicerol foram adquiridos da Sigma (St. Louis, MO). O papel de nitrocelulose (BA85, 0.2ìm) foi da Amersham (Aylesbury, UK). Os anticorpos antiphospho-STAT-1 (sc-7988, goat policlonal) e anti-IFNã (sc-9344, goat policlonal) foram adquiridos da Santa Cruz Biotechnology, Inc. (Santa Cruz, CA).

O sinal foi detectado por meio de reação de quimioluminescência utilizando os reagentes da SuperSignal ${ }^{\circledR W}$ West Pico Chemiluminescent Substrate da Pierce Biothecnology, Inc. Rockford. As bandas autoradiografadas foram quantificadas por meio de densitometria óptica (Gel-Pro Analyzer 3.1 software Exon-Intron Inc., Farrell, MD).

Os resultados foram notificados como média com variação do erro padrão. Utilizou-se análise de variância, seguida de teste de Tukey-Kramer para comparações múltiplas de médias. Nível de significância de $\mathrm{p}<0,05$. 
Rev bras Coloproct

Outubro/Dezembro, 2008

\section{RESULTADOS}

A análise das bandas auto-radiografadas por meio de densitometria óptica mostrou os seguintes dados arbitrários em relação à expressão de IFN- $\gamma$ e à ativação de STAT-1 nos diferentes grupos (RCUI, PAF e Grupo Controle), conforme as figuras ao lado.

A expressão da ativação de STAT-1 foi significativamente maior nos reservatórios ileais dos doentes portadores de RCUI quando comparada a dos Grupos PAF e Controle $(\mathrm{p}<0,05)$. Além disso, os doentes com RCUI apresentaram maior expressão de IFN- $\gamma$ na mucosa do RI, quando comparados ao Grupo Controle $(\mathrm{p}<0,05)$. Apesar de haver uma tendência da expressão de IFN- $\gamma$ ser maior no Grupo RCUI que em PAF, foi considerada similar pelos testes estatísticos ( $p>0.05$ ).

\section{DISCUSSÃO}

A etiologia da ileíte primária do reservatório permanece ainda desconhecida e de certa forma, este fato interfere no aprimoramento e desenvolvimento da profilaxia e tratamento apropriados para esta importante complicação do pós-operatório da cirurgia do RI, principalmente em doentes com RCUI. ${ }^{47,48} \mathrm{O}$ diagnóstico prévio de RCUI parece ser fator de risco, uma vez que a frequiência da "bolsite" é maior entre os doentes portadores de RCUI, quando comparada aos de PAF. ${ }^{37}$ Permanece tópico de discussão se a inflamação da mucosa do RI representa recidiva da RCUI no reservatório, ou seria uma nova forma de doença inflamatória intestinal. ${ }^{35,38}$

A inflamação da mucosa cólica em RCUI envolve elevados níveis de citocinas pró-inflamatórias como IFN- $\gamma$ e de ativadores da transcrição nuclear de TNF- $\alpha$, como STAT- $1 .^{26,28,49}$

As expressões de IFN- $\gamma$ e STAT-1 têm sido verificadas em doenças inflamatórias intestinais, especialmente em RCUI. No entanto, poucos estudos avaliam a atividade imunológica no RI e se há tendência à ativação de vias inflamatórias específicas em mucosa de RI de doentes assintomáticos. Relata-se na literatura, que o desenvolvimento da íleite do RI está relacionado com níveis locais elevados de TNF- $\alpha$, IL-1beta, IL-6 e IL-8. ${ }^{31,32,33,35,36,42}$ Pacientes com PAF, entretanto, demonstram menor expressão de citocinas próinflamatórias, correlacionando com a menor incidência desta complicação pós-operatória neste grupo de doentes. $^{7}$
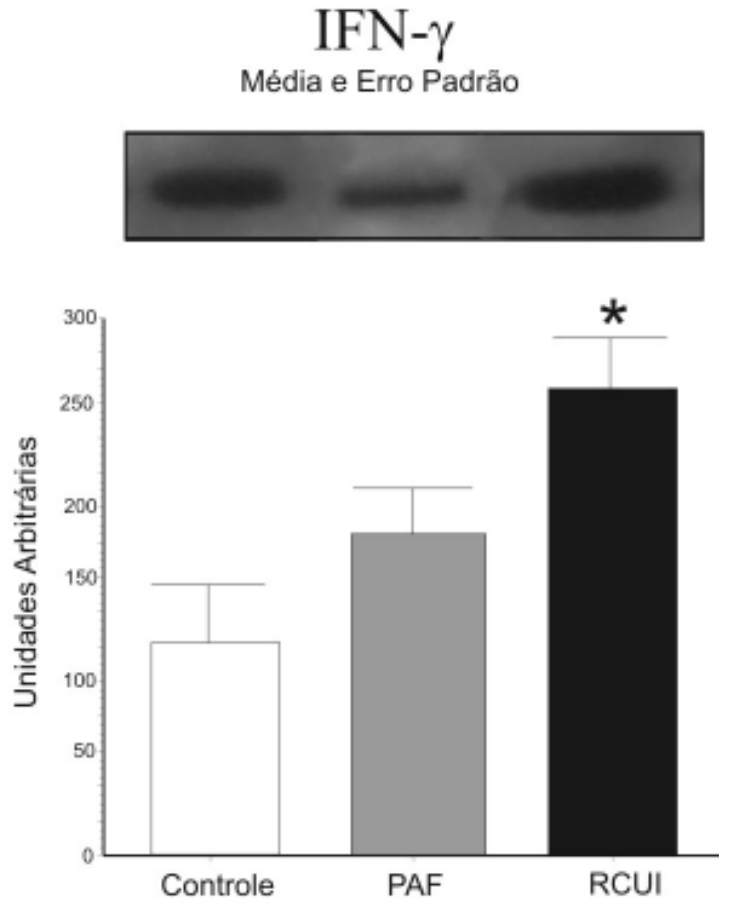

Figura 1 - Expressão de INF- $\gamma$ em mucosa de reservatório ileal normal dos Grupos Controle, PAF e RCUI. Para todas as condições, $n=09$, *p<0.05 vs Control; $\S p<0.05$ vs FAP.

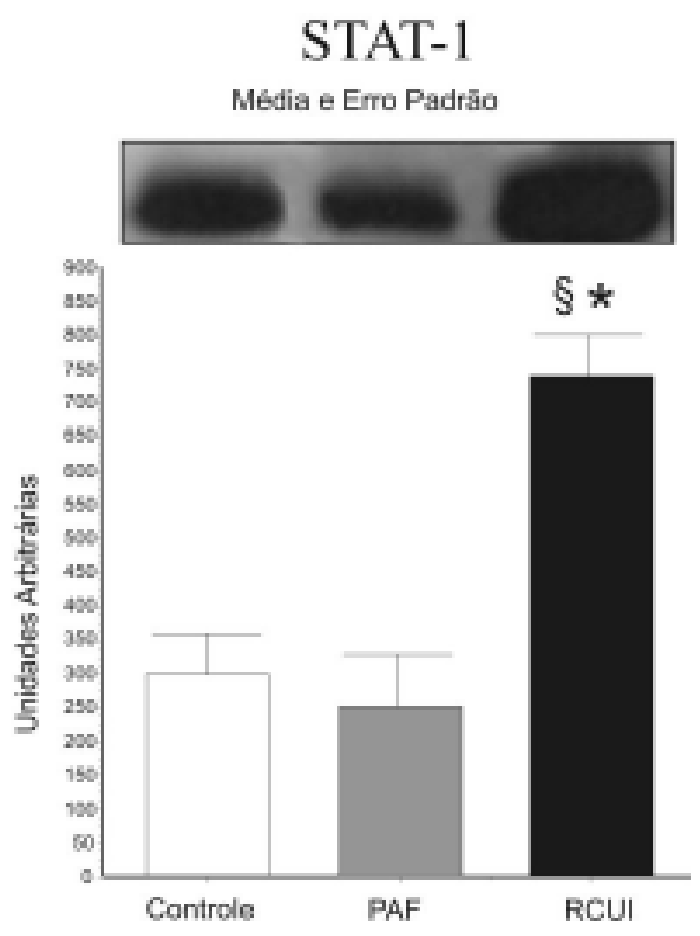

Figura 2 - Ativação de STAT-1 em mucosa de reservatório ileal normal dos Grupos Controle, PAF e RCUI. Para todas as condições, $n=09, * p<0.05$ vs Control; $\S p<0.05$ vs FAP. 
Rev bras Coloproct Outubro/Dezembro, 2008
Apesar da citocina pró-inflamatória TNF- $\alpha$ apresentar importante papel nas vias inflamatórias das doenças inflamatórias intestinais, IFN- $\gamma$ parece estar envolvido de forma mais específica na RCUI. Além disso, STAT-1 e não NF-KB (fator nuclear KB) tem sido associado à principal via responsável de transcrição nuclear do TNF- $\alpha$ em RCUI, sendo ativado pelo IFN- $\boldsymbol{\gamma}^{28,30,50,51}$ Devido a estes fatos, alguns autores têm estudado a expressão de IFN- $\gamma$ e STAT-1 em RI, determinando níveis elevados destas proteínas nas condições de ileíte do RI em portadores de RCUI. ${ }^{37-40}$ Porém, somente um estudo reportou a expressão de IFN- $\gamma$ em RI de PAF. ${ }^{41}$ Nenhum avaliou STAT-1 em PAF.

No presente estudo, avaliou-se a expressão de IFN- $\gamma$ e a ativação do fator de transcrição STAT-1 em mucosa ileal normal de RI de doentes com RCUI e PAF. Observou-se expressão de IFN- $\gamma$ aumentada nos doentes com RCUI quando comparada ao controle de íleo distal normal. Corroborando este achado, a expressão de STAT-1, dependente da citocina IFN- $\gamma$ para sua ativação, foi também maior em RCUI. Este dado é extremamente interessante, pois mesmo em condições clínicas favoráveis, os níveis locais de IFN- $\gamma$ e STAT-1? no RI foram maiores nos portadores de RCUI, quando se comparou à PAF. IFN- $\gamma$, juntamente com TNF- $\alpha$, são conhecidos como citocinas responsáveis pelas fases iniciais de instalação do processo inflamatório, recrutando e ativando monócitos, desta forma, induzindo à expressão de outras citocinas pró-inflamatórias. O fato da expressão de IFN- $\gamma$ não ter sido diferente estatisticamente entre RCUI e PAF, poderia ser explicado pelos doentes serem assintomáticos neste estudo. Além disso, a diferença entre as expressões poderia não ser detectada na ausência de inflamação endoscópica e histológica. Desta forma, constatou-se uma tendência à inflamação nos RI de portadores de RCUI, sendo a via envolvida similar à encontrada na RCUI ativa, podendo explicar a ocorrência maior de "bolsite" nestes casos.

\section{CONCLUSÃO}

Por fim, pode-se demonstrar que apesar das condições clínicas, histológicas e endoscópicas não inflamatórias, os doentes portadores de RCUI operados apresentam atividade inflamatória subclínica nos RI, que é semelhante ao verificado em mucosa cólica de RCUI. Além disso, comparando dois grupos de doentes que se diferenciam quanto à ocorrência de ileíte do RI, pode-se observar as diferenças moleculares no que se refere ao processo inflamatório específico envolvido, aprimorando o conhecimento destas complexas doenças.

\section{AGRADECIMENTOS}

Agradecimentos à enfermeira A. L. N. Domingues do Ambulatório de Doenças Inflamatórias Intestinais - Grupo de Coloproctologia, UNICAMP pelo auxílio técnico, e à Fundação de Amparo à Pesquisa do Estado de São Paulo e ao Fundo de Apoio ao Ensino, Pesquisa e Extensão da UNICAMP pelo auxílio financeiro.

\footnotetext{
ABSTRACT: Pouchitis after total retocolectomy is the most common complication in ulcerative colitis (UC). The immunological mechanisms involved in the genesis of pouchitis are unclear. Purpose: To evaluate STAT-1 activation and IFN-ã expression in normal ileal pouch mucosa. Methods: Eighteen asymptomatic patients submitted to total retocolectomy and $\mathbf{J}$ pouch, were evaluated, being nine with UC and nine with FAP. The activation of STAT-1 and expressions of the cytokine were determined by immunoblot of total protein extracts from pouch mucosal biopsies. Results: STAT-1 activation was increased in UC, when compared to FAP and controls. Higher levels of IFN- $\gamma$ expression were observed in UC when compared to control group, but was similar to FAP. Conclusions: These findings could explain a higher susceptibility to this inflammatory complication in UC when compared to FAP, which may be similar to UC disease.
}

Key Words: Ulcerative colitis; familial adenomatous polyposis, ileal pouch, pouchitis, cytokines.

\section{REFERÊNCIAS}

1. Parks AG, Nicholls RJ. Proctocolectomy without ileostomy for ulcerative colitis. Br Med J 1978;2:85-87

2. Utsunomiya J, Iwama T, Imajo M, et al. Total colectomy, mucosal proctectomy, and ileoanal anastomosis. Dis Colon Rectum 1980;23:459-466
3. Nicholls RJ, Moskovitz RL, Shepard NA. Restorative proctocolectomy with ileal reservoir. Br J Surg 1985;72:76-79

4. Nicholls RJ. Restorative proctocolectomy with various types of reservoir. World J Surg 1987;11:751-762

5. M'Koma AE. Serum biochemical evaluation of patients with functional pouches tem to 20 years after restorative proctocolectomy. Int J Colorectal Dis 2006;26:1-10 
6. Mc Guire BB, Brannigan AE, O'Connell PR. Ileal pouch-anal anastomosis. Br J Surg 2007;94:812-23

7. Kiehne K, Brunke G, Wegner F, et al. Defensin expression in chronic pouchitis in patients with ulcerative colitis or familial adenomatous polyposis coli. World J Gastroenterol 2006;21:12(7):1056-1062

8. Heuschen UA, Allemeyer EH, Hinz U, et al. Diagnosing pouchitis: comparative validation of two scoring system in routine follow-up. Dis Colon Rectum 2002;45(6):776-786

9. Shen B, Fazio VW, Renzi FH, et al. Risk factors for disease of ileal pouch-anal anastomosis after restorative proctocolectomy for ulcerative colitis. Clin Gastroenterol Hepatol 2006;4(1):8189

10. Moskowitz RL, Shepard NA, Nicholls RJ. An assessment of inflammation in the reservoir after restorative proctocolectomy with ileoanal ileal reservoir. Int J Colorectal Dis 1986;1:167174

11. Meagher AP, Farouk R, Dozois RR, et al. J ileal pouch-anal anastomosis for chronic ulcerative colitis: complications and long-term outcome in 1310 patients. Br J Surg 1998;85:800803

12. Armstrong DN, Sillin LF, Chung R. Reduction in tissue blood flow in J-shaped pelvic ileal reservoirs. Dis Colon Rectum 1995;38:526-529

13. Laake KO, Bjorneklett A, Aamodt G, et al. Outcome of four weeks's intervention with probiotics on symptoms and endoscopic appearance after surgical reconstruction with a Jconfigurated ileal-pouch-anal-anastomosis in ulcerative colitis. Scand J Gastroenterol 2005;40:43-51

14. Ohge H, Furne JK, Springfield J, et al. Association between fecal hydrogen sulfide production and pouchitis. Dis Colon Rectum 2005;48:469-475

15. Kuehbacher T, Ott SJ, Helwig U, et al. Bacterial and fungal microbiota in relation to probiotic therapy in pouchitis. Gut 2006;55(6):833-841

16. Sandborn WJ. Pouchitis following ileal pouch anal anastomosis: definition, pathogenesis, and treatment. Gastroenterology 1994;107:1856-1860

17. Nicholls RJ, Banerjee AK. Pouchitis: risk factores, etiology, and treatment. World J Surg 1998;22:347-356

18. Kienle P, Weitz J, Reinshagen S, et al. Association of decreased perfusion of the ileoanal pouch mucosa with early postoperative pouchitis and local septic complications. Arch Surg 2001;136:1124-30

19. Heuschen G, Leowardi C, Hinz U, et al. Differential expression of toll-like receptor 3 and 5 in ileal pouch mucosa of ulcerative colitis patients. Int J Colorectal Dis 2007;22:293-301

20. Sandborn WJ, Tremaine WJ, Batts KP, et al. Pouchitis after ileal pouch-anal anastomosis: a Pouchitis disease activity Index. Mayo Clin Proc 1994;69:409-415

21. Shen B, Achkar JP, Connor JT, et al. Modified Pouchitis Disease Activity Index. A simplified approach to the diagnosis of pouchitis. Dis Colon Rectum 2003;46(6):748-753
22. Akerlung JE, Lofberg R. Pouchitis. Curr Opin Gastroenterol 2004;20(4):341-344

23. Lohmuller JL, Pemberton JH, Dozois RR, et al. Pouchitis and extraintestinal manifestations of inflammatory bowel disease after ileal pouch-anal anastomosis. Ann Surg 1990;211(5):622629

24. Hata K, Watanabe T, Shinozaki M, et al. Patients with extraintestinal manifestations have a higher risk of developing pouchitis in ulcerative colitis: multivariate analysis. Scand $\mathbf{J}$ Gastroenterol 2003;38:1055-1058

25. O'Shea J, Ma A, Lipsky P. Cytokines and autoimmunity. Nature Reviews 2002;2:37-45

26. Mudter J, Weigmann B, Bartsch B, et al. Activation pattern of signal transducers and activators of transcription (STAT) factors in inflammatory bowel disease. Am J Gastroenterol 2005;100:64-72

27. Pullman WE, Elsbury S, Kobayashi M, et al. Enhanced mucosal cytokine production in inflammatory bowel disease. Gastroenterology 1992;102:529-537

28. Schreiber S, Rosenstiel P, Hampe J, et al. Activation of signal tranducer and activator of transcription (STAT) 1 in human chronic inflammatory bowel disease. Gut 2002;51:379-385

29. Klampfer L, Huang J, Sasazuki T, et al. Inhibition of Interferon $\gamma$ signaling by the short chain fatty acid butyrate. Mol Cancer Res 2003;1:855-62

30. Schreiber S, Hampe J, Nikolaus S, et al. Patterns of cytokine transcription factor activation are different in Crohn's disease (CD) and ulcerative colitis (UC). Gastroenterology 1997;112(4):A1086

31. Gionchetti P, Campieri M, Belluzzi A, et al. Mucosal concentrations of interleukin-1 beta, interleukin-6, interleukin8 , and tumor necrosis factor-alpha in pelvic ileal pouches. Dig Dis Sci 1994;39:1525-1531

32. Evgenikos N, Bartolo DCC, Hamer-Hodges DW, et al. Assessment of ileoanal pouch inflammation by interleukin 1â and interleukin 8 concentrations in the gut lumen. Dis Colon Rectum 2002;45:249-255

33. Bulois P, Tremaine WJ, Maunoury V, et al. Pouchitis is associated with mucosal imbalance between interleukin- 8 and interleukin-10. Inflamm Bowel Dis 2000;6:157-167

34. Lammers KM, Vergopoulos A, Babel N, et al Probiotic therapy in the prevention of pouchitis onset: decreased Interleukin1â, Interleukin-8 and Interferon-ã gene expression. Inflamm Bowel Dis 2005;11(5):447-454

35. Schmidt C, Giese T, Ludwig B, et al. Increased cytokine transcripts in pouchitis reflect the degree of inflammation but not the underlying entity. Int J Colorectal Dis 2006;21:419426

36. Helwig U, Gionchetti P, Rizello F, et al. CXC and CC chemokine expression in inflamed and noninflamed pelvic ileal pouch tissue. Int J Colorectal Dis 2004;19:165-170

37. Ulisse S, Gionchetti P, D'Aló S, et al. Expression of cytokines, inducible nitric oxide synthase, and matrix metalloproteinases 
in pouchitis: Effects of probiotic treatment. Am J Gastroenterol 2001;96(9):2691-9

38. Kuhbacher T, Gionchetti P, Hampe J, et al. Activation of signal-transducer and activator of transcription 1(STAT1) in pouchitis. Clin Exp Immunol 2001;123:395-401

39. Boerr L, Sambuelli A, Sugay E, et al. Serum TGF-âl and Interferon -ã levels in ileal pouch-anal anastomosis in ulcerative colitis. Gastroenterology 1998;114:A936

40. Schreiber S, Gionchetti P, Hampe J, et al. Pouchitis shares similarities with ulcerative colitis in the pattern of cytokine transcription factor activation. Gastroenterology 1997;112(4):A1086

41. Stallmach A, Schafer F, Hoffmann S, et al. Increased state of activation of CD4 positive $\mathrm{T}$ cells and elevated interferon a production in pouchitis. Gut 1998;43:499-505

42. Patel RT, Bain I, Youngs D, et al. Cytokine production in pouchitis is similar to that in ulcerative colitis. Dis Colon Rectum 1995;38:831-837

43. Góes JRN, Coy CSR, Amaral CA, et al. Superior mesenteric artery symdrome as a complication of ileal pouch-anal anastomosis. Dis Colon Rectum 1995;38:543-4

44. Bradford MM. A rapid and sensitive method for the quantitation of microgram quantities of protein utilizing the principle of protein-dye binding. Anal Biochem 1976;72:248254
45. Velloso LA, Folli F, Sun XJ, et al. Cross-talk between the insulin and angiotensin signaling systems. Proc Natl Acad Sci USA 1996;93:12490-12495

46. Araújo EP, De Souza CT, Gasparetti AL, et al. Short-term in vivo inhibition of insulin receptor substrate-1 expression leads to insulin resistence, hyperinsulinemia, and increased adiposity. Endocrinology 2005;146:1428-1437

47. Kuhbacher T, Schreiber S, Runkel N. Pouchitis: pathophysiology and treatment. Int J Colorectal Dis 1998;13:196-207

48. Tulchinsky H, Hawley PR, Nicholls J. Long-term failure after restorative proctocolectomy for ulcerative colitis. Ann Surg 2003;238(2):229-234

49. Kuhn K, Lohler J, Rennick D, et al. Interleukin-10-deficient mice develop chronic enterocolitis. Cell 1993;75:263-74

50. Chen LF, Greene WC. Shaping the nuclear action of NF-B. Nature Reviews 2004;5:392-401

51. Schreiber S, Nikolaus S, Hampe J. Activation of nuclear factor KB in inflammatory bowel disease. Gut 1998;42:477-84.

\section{Endereço para correspondência:}

RAQUEL FRANCO LEAL

Rua Patativa, $\mathrm{n}^{\circ} 170$, apto $24 \mathrm{C}$

Bonfim - CEP 13034-810

Campinas, São Paulo

Tel. -19-32844803

raquelleal@mpc.com.br

ccoy@terra.com.br 